\title{
Flexibility in the timeline of early eukaryote evolution
}

\author{
SUSANNAH PORTER ${ }^{1}$, DR. HEDA AGIĆ ${ }^{1}$, PHOEBE
} COHEN $^{2}$ AND CHRISTOPHER JUNIUM ${ }^{3}$

${ }^{1}$ University of California Santa Barbara

${ }^{2}$ Williams College

${ }^{3}$ Syracuse University

Presenting Author: porter@geol.ucsb.edu

The origin and early evolution of eukaryotes is one of the most important events shaping Earth's history. While the broad timeline is known, there is significant flexibility in how it might be interpreted. The oldest widely accepted eukaryote fossils are ornamented vesicles in rocks $1.65 \mathrm{Ma}$. These represent the first appearance of diagnosably eukaryotic characters in the clade, but do not record the origin of the group. Rather, recent estimates suggest the first eukaryotic common ancestor (FECA) appeared in the early Paleoproterozoic (c. $2.3 \mathrm{Ga}$ ), suggesting hundreds of millions of years during which stem eukaryotes were present and potentially among the fossils preserved from this time but just aren't recognized. The oldest widely accepted evidence of crown group eukaryotes occur in rocks ca. 1.0-1.1 Ga; estimates for the appearance of the last eukaryotic common ancestor (LECA) range widely, but permit the possibility of an origin close to 1.0 $1.1 \mathrm{Ga}$. If true, it implies a long interval between FECA and LECA during which the characters that define modern eukaryotes appeared. This has important implications for how the early eukaryotic record is interpreted: because we don't know the order in which defining eukaryotic characters evolved, there are several possible narratives of early eukaryote evolution and its link to environmental change. A widely accepted narrative assumes very early acquisition of aerobic metabolism and steranes (shortly after FECA), implying that virtually all younger eukaryotes were aerobic and sterol-producing. The absence of steranes from Mesoproterozoic and older rocks would therefore reflect either taphonomic artefact or suppressed abundance of eukaryotes because of unfavorable conditions (e.g. widespread anoxia). An equally permissible endmember narrative, however, is that aerobic metabolism and sterols evolved very late (e.g. 1.1 Ga). This would imply that no older eukaryotes were aerobic or made (diagnostically eukaryotic) sterols; that the absence of steranes in rocks $>1.1$ Ga reflects real absence; that older eukaryotes were not restricted to, and therefore not proxies for, oxic settings; and that the GOE did not drive eukaryote evolution. I will discuss ways in which we might distinguish among these possibilities. 